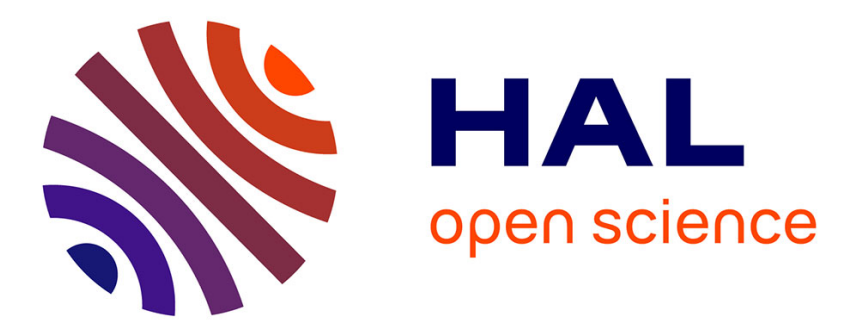

\title{
Numerical and experimental study of parabolic pulses generated via Raman amplification in standard optical fibers
}

Christophe Finot, Guy Millot, Stéphane Pitois, Cyril Billet, John M Dudley

\section{- To cite this version:}

Christophe Finot, Guy Millot, Stéphane Pitois, Cyril Billet, John M Dudley. Numerical and experimental study of parabolic pulses generated via Raman amplification in standard optical fibers. IEEE Journal of Selected Topics in Quantum Electronics, 2004, 10 (5), pp.1211-1218. 10.1109/JSTQE.2004.837214 . hal-00095483

\section{HAL Id: hal-00095483 \\ https://hal.science/hal-00095483}

Submitted on 25 Mar 2021

HAL is a multi-disciplinary open access archive for the deposit and dissemination of scientific research documents, whether they are published or not. The documents may come from teaching and research institutions in France or abroad, or from public or private research centers.
L'archive ouverte pluridisciplinaire $\mathbf{H A L}$, est destinée au dépôt et à la diffusion de documents scientifiques de niveau recherche, publiés ou non, émanant des établissements d'enseignement et de recherche français ou étrangers, des laboratoires publics ou privés. 


\title{
Numerical and Experimental Study of Parabolic Pulses Generated via Raman Amplification in Standard Optical Fibers
}

\author{
Christophe Finot, Guy Millot, Stéphane Pitois, Cyril Billet, and John M. Dudley, Member, IEEE
}

\begin{abstract}
Parabolic pulse generation by Raman amplification has been numerically and experimentally investigated around $1550 \mathrm{~nm}$ using a standard normally dispersive nonzero dispersion shifted fiber (NZ-DSF). The output pulses, characterized in intensity and phase using frequency-resolved optical gating, exhibit parabolic features in good agreement with numerical simulations based on two coupled extended nonlinear Schrödinger equations. The influence of the energy and duration of the input pulse has been studied. The ability of the parabolic pulses to propagate self-similarly during additional propagation over 800 $m$ of NZ-DSF has also been demonstrated.
\end{abstract}

Index Terms-Frequency-resolved optical gating (FROG) characterization, nonlinear fiber optics, optical self-similarity, parabolic pulses, pulse compression, pulse shaping, ultrashort pulse amplification.

\section{INTRODUCTION}

$\mathbf{P}$ ARABOLIC pulses in normally dispersive optical fibers with gain have generated considerable interest since their first experimental demonstration [1]. Theoretical studies [2], [3] based on the self-similarity analysis of the nonlinear Schrödinger equation (NLSE) with constant gain, have revealed that the interplay of normal dispersion, nonlinearity, and gain produces a linearly chirped pulse with a parabolic intensity profile which resists the deleterious effects of optical wave-breaking [4]. Parabolic pulses represent a new class of solution to the NLSE, as they are generated asymptotically in the fiber amplifier independently of the shape or noise properties of the input pulse, and propagate self-similarly subject to exponential scaling of amplitude and temporal width. To date, experimental studies of parabolic pulse generation have been restricted to fiber amplifiers where the gain and normal group velocity dispersion (GVD) are associated with the addition of dopants, such as Erbium [1] or Ytterbium [5]-[7], in the fiber core. These studies have also shown the wide-ranging practical

C. Finot, G. Millot, and S. Pitois are with the Laboratoire de Physique de l'Université de Bourgogne, Centre National de la Recherche Scientifique, 21078 Dijon, France (e-mail: guy.millot@u-bourgogne.fr).

C. Billet and J. M. Dudley are with the Laboratoire d'Optique P. M. Duffieux de l'Université de Franche-Comté, Centre National de la Recherche Scientifique, 25030 Besançon, France (e-mail: John.Dudley@univ-fcomte.fr). significance of parabolic pulses associated with the efficient compensation of their linear chirp, which can lead to high quality compressed pulses. The inclusion of parabolic pulses technology in femtosecond fiber lasers has also demonstrated theoretically and experimentally the ability to increase the pulse energy by two orders of magnitude [8].

It should be noted that the fiber gain mechanism that is used for the parabolic pulse generation need not necessarily be based on a resonant amplification process, and this opens up the possibility to consider the use of Raman amplifiers to provide gain where no conventional amplifiers are possible. Moreover, this allows us to take advantage of their broad amplification bandwidths which can exceed $100 \mathrm{~nm}$. Indeed, numerical simulations [9] have shown that a properly designed photonic crystal fiber (PCF) pumped with high power nanosecond pulses can indeed support the generation of parabolic pulses through Raman amplification. More recent numerical [10] and experimental [11] studies have relaxed the constraint on the fiber type required for this purpose, showing that Raman parabolic pulse generation is possible using only standard normally dispersive nonzero dispersion shifted fiber (NZ-DSF) at $1550 \mathrm{~nm}$ in association with a commercial watt-level continuous-wave $(\mathrm{CW})$ pump source.

In this paper, we present a detailed description of both the experimental and numerical studies that have considered Raman parabolic pulse generation in normally dispersive NZ-DSF at $1550 \mathrm{~nm}$. The paper is organized as follows. In Section II, we describe numerical simulations of the Raman parabolic pulse amplification process that were used in the design of our experiments. We focus our attention on walk-off effects between the signal and pump waves and discuss a computationally-efficient model based on two coupled extended NLSEs, rather than by a single extended scalar NLSE. In Section III, we describe our experimental Raman amplifier and pulse characterization setup, and we present results that experimentally verify the generation of linearly chirped parabolic pulses at the Raman amplifier output. Section IV presents results of additional experiments studying the effect of the input pulse duration and energy on the amplified parabolic pulses, and we also experimentally verify the ability of parabolic pulses to propagate in a passive normally dispersive fiber while maintaining their linear chirp. Finally, we show numerically that parabolic pulses can lead, after linear compensation of their chirp, to high-quality chirp-free pulses. Section V concludes the paper. 


\section{NUMERICAL SIMULATIONS}

\section{A. Scalar Extended NLSE}

Most previous studies of parabolic pulses have been largely restricted to the case where pulse propagation is accurately modeled by the addition of a simple constant gain to the standard NLSE. In this context, analytical exact asymptotic self-similar solutions in the limit $z \rightarrow \infty$ (where $z$ represents the propagation coordinate) have been described [3]. Some work has also been carried out to investigate the effects of a longitudinally varying gain profile [3], as well as the limiting effects of the finite transition linewidth [12]. In order to investigate the possibility of Raman parabolic pulse generation, we use a numerical model based on the extended NLSE [13]

$$
\begin{aligned}
& i \frac{\partial A}{\partial z}=-i \frac{\alpha}{2} A+\frac{\beta_{2}}{2} \frac{\partial^{2} A}{\partial t^{2}}+i \frac{\beta_{3}}{6} \frac{\partial^{3} A}{\partial t^{3}} \\
&-\gamma\left[1+\frac{i}{\omega_{s}} \frac{\partial}{\partial t}\right] A(z, t) \int_{0}^{\infty} R\left(t^{\prime}\right)\left|A\left(z, t-t^{\prime}\right)\right|^{2} d t^{\prime}
\end{aligned}
$$

Here, $A(z, t)=A_{s}(z, t)+A_{p}(z, t) \exp (-i \Omega t)$, where $A_{s}(z, t)$ and $A_{p}(z, t)$ are slowly varying envelopes for signal and pump fields oscillating at $\omega_{s}$ and $\omega_{p}$, and $\Omega=\omega_{p}-\omega_{s}$ is the pumpsignal frequency detuning. The inclusion of the time-derivative operator $\left(i / \omega_{s}\right) \partial / \partial t$ in the nonlinear term is necessary to ensure that the photon number is conserved, and not the optical energy, so that the Raman interaction is described correctly. The function $R(t)=\left(1-f_{r}\right) \delta(t)+f_{r} h_{r}(t)$ includes the instantaneous electronic response and the delayed Raman contribution, with the fractional Raman contribution $f_{r}=0.18$. For $h_{r}(t)$, we used the measured Raman response of fused silica [14].

The dashed line (right axis) in Fig. 1 shows the imaginary part of $h_{r}(t)$, compared with a typical experimental spectrum of a parabolic pulse obtained with our parameters described later, shown as the solid line (left axis). These results are shown at this stage in order to illustrate that for our choice of parameters, the amplified spontaneous Raman emission intensity is $20 \mathrm{~dB}$ lower than that of the amplified signal pulse, and thus we are justified in neglecting it in our numerical simulations. We also note that the output pulse spectrum is significantly narrower than the Raman gain bandwidth so that we can expect that bandwidth limiting (solitary wave) effects are negligible [12]. The experimental spectrum also shows that achieving peak Raman gain at $1550 \mathrm{~nm}$ requires a CW pump at a wavelength of $1455 \mathrm{~nm}$.

The Raman amplifier considered in this work is based on 5.3 $\mathrm{km}$ of NZ-DSF with dispersion parameters at $1550 \mathrm{~nm}$ corresponding to GVD parameter $\beta_{2}=4.89 \times 10^{-3} \mathrm{ps}^{2} \mathrm{~m}^{-1}$ and a third-order dispersion $\beta_{3}=1.09 \times 10^{-4} \mathrm{ps}^{3} \mathrm{~m}^{-1}$, so that the fiber is normally dispersive at the wavelength of the input pulses which are being amplified. The nonlinearity coefficient used is $\gamma=2.23 \times 10^{-3} \mathrm{~W}^{-1} \mathrm{~m}^{-1}$. The wavelength dependent loss coefficient $\alpha$ is also included in (1). The input pulse initial conditions $A_{s}(0, t)$ correspond to the intensity and chirp of our 1550 -nm pulses, which were measured experimentally using the second-harmonic generation frequency-resolved optical gating (SHG-FROG) technique described in Section III. We typically used signal pulses with durations in the range of 7-14 ps, and with energies in the range $0.5-3 \mathrm{pJ}$.

We use the standard split-step Fourier method [13] to solve (1). Preliminary simulations have previously indicated that, with

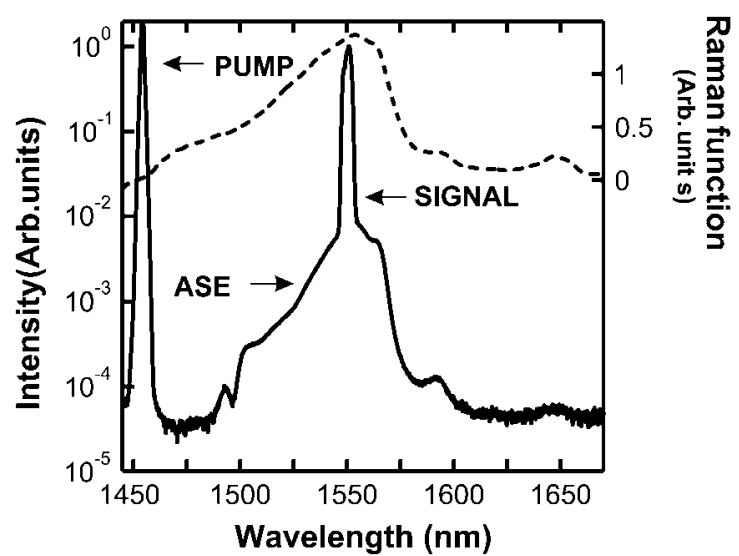

Fig. 1. Experimental spectrum (solid line, left axis) of the output pulse after $5.3 \mathrm{~km}$ of propagation in a NZ-DSF, with a gain of $18.8 \mathrm{~dB}$. The initial pulse was a 3-pJ and 10-ps pulse at $1550 \mathrm{~nm}$. Imaginary part (dashed line, right axis) of the Raman function $h_{r}$ due to the pump at $1455 \mathrm{~nm}$.

the parameters described earlier, a propagation distance of several kilometers is required to enter into the parabolic pulse amplification regime [10]. This propagation distance is large compared to lengths ( $\sim$ meters) required in the case of doped fibers [1]-[6] or PCF [9] where the distributed gain per unit length is significantly higher. The walk-off effects due to the groupvelocity mismatch between the pump and signal waves must, therefore, be accurately taken into account. The walk-off parameter is defined as $\delta=\beta_{1 \mathrm{p}}-\beta_{1 \mathrm{~s}}$ where $\beta_{1}=d \beta / d \omega$ with $\beta$ the propagation constant and the subscripts $p$ and $s$ are relative to pump and signal waves, respectively. For the NZ-DSF considered in our experiments, $\delta=1.07 \mathrm{ps} \mathrm{m}^{-1}$ so that in order to obtain accurate numerical solutions of (1) over a $5.3 \mathrm{~km}$ propagation distance, a minimum temporal window of $6 \mathrm{~ns}$ is required. Since accurate modeling of both the pump and signal fields over the Raman bandwidth requires a spectral window of at least 50 $\mathrm{THz}$ (see Fig. 1), the Fourier discretization of the temporal and spectral spaces requires typically $2^{18}$ points and leads to prohibitively long computation times. This problem can, however, be conveniently avoided by the separate modeling of the pump and signal evolution as considered in the following subsection.

\section{B. Coupled Extended Nonlinear Schrödinger Equations}

The propagation of two copropagating frequencies in an optical fiber can be described by two incoherently coupled NLSEs, whenever the four-wave interaction between the two input waves can be neglected. In this case, the extended scalar NLSE [see (1)], may be reduced to the following set of two incoherently coupled extended NLSE's [15]:

$$
\begin{aligned}
i \frac{\partial A_{s}}{\partial z} & =-i \frac{\alpha_{s}}{2} A_{s}+\frac{\beta_{s 2}}{2} \frac{\partial^{2} A_{s}}{\partial t^{2}}+i \frac{\beta_{s 3}}{6} \frac{\partial^{3} A_{s}}{\partial t^{3}} \\
& -\gamma_{s}\left[1+\frac{i}{\omega_{s}} \frac{\partial}{\partial t}\right]\left\{\left(1-f_{r}\right) A_{s}\left(\left|A_{s}\right|^{2}+2\left|A_{p}\right|^{2}\right)\right. \\
& +f_{r} A_{s} \int_{0}^{\infty} h\left(t^{\prime}\right)\left(\left|A_{s}\left(z, t-t^{\prime}\right)\right|^{2}\right. \\
& \left.+\left|A_{p}\left(z, t-t^{\prime}\right)\right|^{2}\right) d t^{\prime} \\
& \left.+f_{r} A_{p} \int_{0}^{\infty} h\left(t^{\prime}\right) A_{s}\left(z, t-t^{\prime}\right) A_{p}^{*}\left(z, t-t^{\prime}\right) e^{-i \Omega t} d t^{\prime}\right\}
\end{aligned}
$$



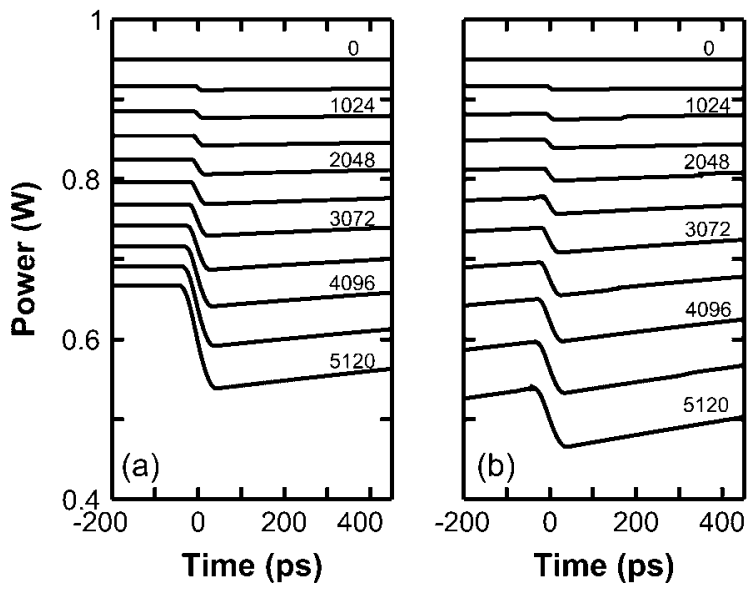

Fig. 2. Evolution of the pump field and walk-off effects during propagation in a $5.3 \mathrm{~km}$ NZ-DSF for an initial 10-ps and 3-pJ input pulse. (a) Simulations carried out from coupled NLSEs [(2)] with an adequate temporal window. (b) Simulations based on the scalar NLSE [(1)] using an inadequate temporal window of $500 \mathrm{ps}$, showing artificial pump depletion on the left side. The time origin corresponds to the position of the signal pulse.

$$
\begin{aligned}
i \frac{\partial A_{p}}{\partial z} & =-i \frac{\alpha_{p}}{2} A_{p}-i \delta \frac{\partial A_{p}}{\partial t}+\frac{\beta_{p 2}}{2} \frac{\partial^{2} A_{p}}{\partial t^{2}}+i \frac{\beta_{p 3}}{6} \frac{\partial^{3} A_{p}}{\partial t^{3}} \\
& -\gamma_{p}\left[1+\frac{i}{\omega_{p}} \frac{\partial}{\partial t}\right]\left\{\left(1-f_{r}\right) A_{p}\left(\left|A_{p}\right|^{2}+2\left|A_{s}\right|^{2}\right)\right. \\
& +f_{r} A_{p} \int_{0}^{\infty} h\left(t^{\prime}\right)\left(\left|A_{s}\left(z, t-t^{\prime}\right)\right|^{2}\right. \\
& \left.+\left|A_{p}\left(z, t-t^{\prime}\right)\right|^{2}\right) d t^{\prime} \\
& \left.+f_{r} A_{s} \int_{0}^{\infty} h\left(t^{\prime}\right) A_{p}\left(z, t-t^{\prime}\right) A_{s}^{*}\left(z, t-t^{\prime}\right) e^{i \Omega t} d t^{\prime}\right\}
\end{aligned}
$$

The important approximation here of neglecting four-wave mixing coherent terms and higher-order Raman effects is justified by the absence of any such spectral components in the results shown in Fig. 1. Note that the last three terms on the right-hand side of (2) correspond to the Raman-like terms which represent the self-frequency shift, the cross-frequency shift, and the amplification (or depletion), respectively. Within the experimental conditions depicted in our paper, Raman self- and cross-frequency shifts play a negligible role for the parabolic pulse generation compared to the effects of Raman amplification or depletion. We have checked from numerical integration of (2) that cancellation of the frequency shift terms would indeed lead to variations of the peak power and pulse width of the amplified pulse less than $4 \%$.

When solving these coupled equations using the split-step Fourier method, a spectral window of only a few terahertz (adapted to the spectral width of the output pulse) can be used, resulting in orders of magnitude improvements in computation time.

Fig. 2(a) shows the depletion effect of the pump wave as a function of the distance of propagation, as calculated from numerical integration of (2). Note that, according to (2), the signal pulse remains very close to $t=0$ during its propagation through the fiber. On the other hand, as can be seen in Fig. 2(a), the walk-off effect between the pump and signal waves leads to a continuous temporal shift of the pump wave with respect to the temporal position of the signal pulse, as the propagation distance increases. This temporal shift leads to a regeneration of the pump, and to a larger efficiency of the self-similar transformation of the input signal pulse. Indeed, the decrease of the pump power at the raising edge of the pulse is just due to the linear attenuation of the fiber. For comparison, a similar analysis has been done from numerical integration of (1), by considering only a 500-ps temporal discretization window to reduce the computation time. The corresponding numerical results, reported in Fig. 2(b), clearly evidence a nonphysical excess of pump depletion due to the periodic nature of the fast-Fourier-transform algorithm.

As regard to the earlier considerations, in the following part of this paper, we only consider numerical integrations of the two coupled NLSEs [(2)].

\section{EXPERIMENTAl SETUP AND PARABOLIC NATURE OF AMPLIFIED PULSES}

\section{A. Experimental Setup and Typical FROG Traces}

Fig. 3(a) shows a schematic representation of the experimental setup used. The $1550-\mathrm{nm}$ input pulses are obtained from a $22-\mathrm{MHz}$ repetition rate Pritel FFL passively mode-locked fiber laser that allowed for the possibility of generating pulses of variable duration, typically a few picoseconds. The energy of the signal pulses can be also adjusted, and in the following, we have mainly used pulses with an energy of $2.16 \mathrm{pJ}$. The Raman gain is provided by a CW Keopsys 2-W Raman laser at $1455 \mathrm{~nm}$. The pump power at the laser output is fixed to 1.75 W but, taking into account losses introduced by connectors and wavelength-division multiplexing (WDM) couplers, the input power injected into the NZ-DSF fiber is only $1.3 \mathrm{~W}$. A WDM coupler with high power ratings permits the superposition of pump and signal beams in the $5.3 \mathrm{~km}$ of commercial NZ-DSF. Another coupler at the fiber output isolates the amplified signal pulse with a rejection level of $21 \mathrm{~dB}$.

Input and output pulses are characterized by the SHG-FROG device shown in detail in Fig. 3(b). Fiber connectorized components are used to divide the input beam into two separated beams and to introduce an optical time delay before noncollinear Type I SHG in a BBO crystal. The SHG spectrum is measured by an ANRITSU MS 9710B optical spectrum analyzer (OSA). The phase and intensity retrieval was performed using the generalized projections algorithm. The fidelity on the FROG measurements was checked using the standard techniques based on comparisons of the independently-measured autocorrelation and spectrum with the FROG trace marginals [16].

Fig. 4 shows typical SHG-FROG traces of input (a) and output (b) pulses in the case of an initial near transform-limited sech $^{2}$ intensity profile, with 7-ps full-width at half-maximum (FWHM), 2.16-pJ pulse energy and 230-mW peak power. Fig. 4 clearly shows significant spectral and temporal broadenings of the pulse after propagation through the fiber, obtained with a Raman amplification gain of $18.7 \mathrm{~dB}$. The retrieval error of the output pulse is typically less than $9 \times 10^{-3}$ with a $1024 \times 1024$ grid. We note in this context that retrieval errors 


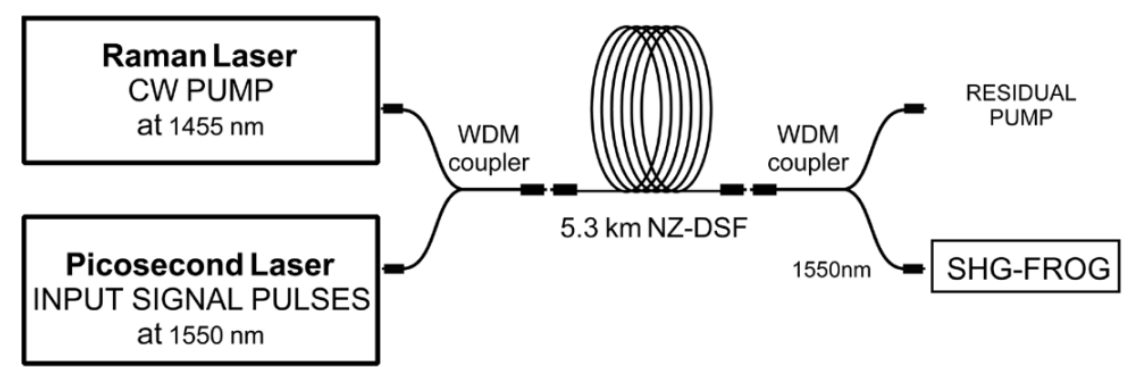

(a)

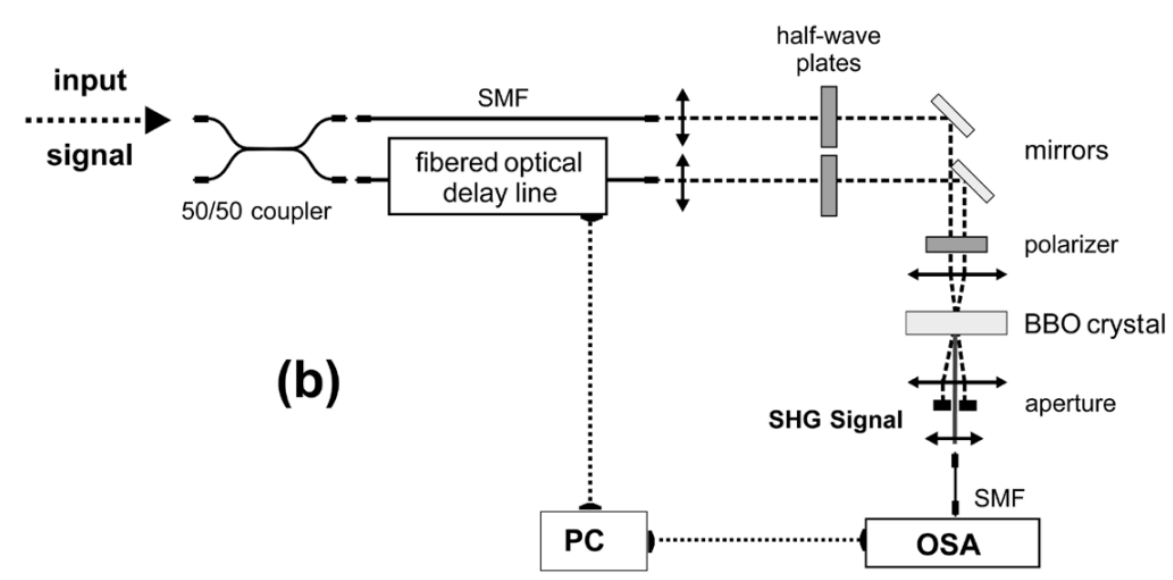

Fig. 3. Schematic diagram of experimental setups. (a) Setup of the Raman amplifier used to generate parabolic pulses. (b) Setup of the SHG-FROG device.

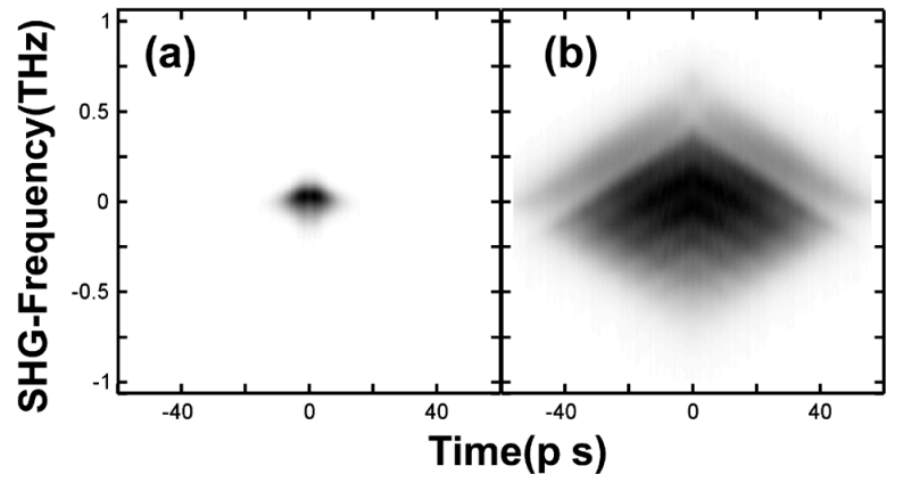

Fig. 4. Typical measured FROG traces of (a) an input pulse of 6-ps FWHM temporal width and 2.16-pJ energy and of (b) the corresponding output pulse after propagation in a 18.7-dB Raman amplifier based on $5.3 \mathrm{~km}$ of NZ-DSF.

for highly chirped pulses must be interpreted in light of the significant nonzero data fraction of the trace, which is defined in terms of the fraction of the data having an intensity greater than $1 \%$ of the trace maximum. For the output FROG traces we study, the nonzero data fraction is only around $15 \%$ so that these retrieval errors are acceptably low [16].

\section{B. Parabolic Nature of Amplified Pulses}

Fig. 5 shows the intensity and chirp retrieved from FROG measurement of the output pulse (open circles). The ouput pulse peak power is $3.3 \mathrm{~W}$ and its width is $46 \mathrm{ps}$ (at FWHM) and $72 \mathrm{ps}$ at the $-20 \mathrm{~dB}$ points (this latter measure of pulse duration is particularly useful for parabolic pulses in view of the rapid intensity falloff in the wings). The output pulse intensity profile as well as the pulse chirp are, respectively, compared

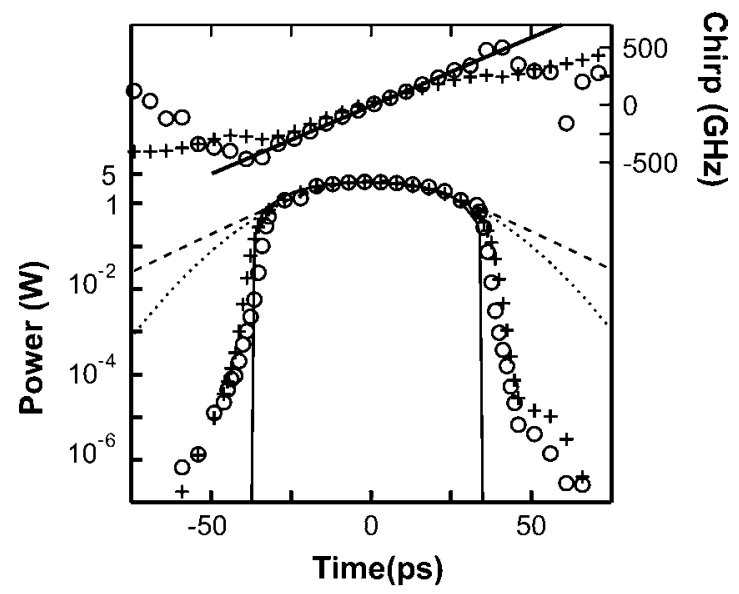

Fig. 5. Intensity and chirp profiles of output pulse after $5.3 \mathrm{~km}$ of propagation of 7-ps and 2.16-pJ input pulse. Results from FROG trace retrieval (circles) compared with numerical simulations (crosses). Linear and parabolic fit (solid lines) of, respectively, chirp and intensity profiles. Gaussian (dashed line) and $\operatorname{sech}^{2}$ (dotted line) fits illustrating the comparatively poor fits obtained using these pulse shapes compared to a parabolic pulse.

with least-squares parabolic and linear fits shown as solid lines in the figure. The good fits obtained illustrate clearly the linearly-chirped parabolic nature of pulses at the Raman amplifier output. For comparison, Fig. 5 also includes Gaussian (dotted line) and $\operatorname{sech}^{2}$ (dashed line) fits to illustrate the much-improved goodness of fit that is obtained using a parabolic pulse profile, especially regarding the characteristic rapid falloff in the wings. Let us remark that the intensity profile of the amplified pulse exhibits a small asymmetry, which can be in part explained by the slight asymmetry of the initial pulse (not shown here). However, numerical simulations carried out with symmetric input 


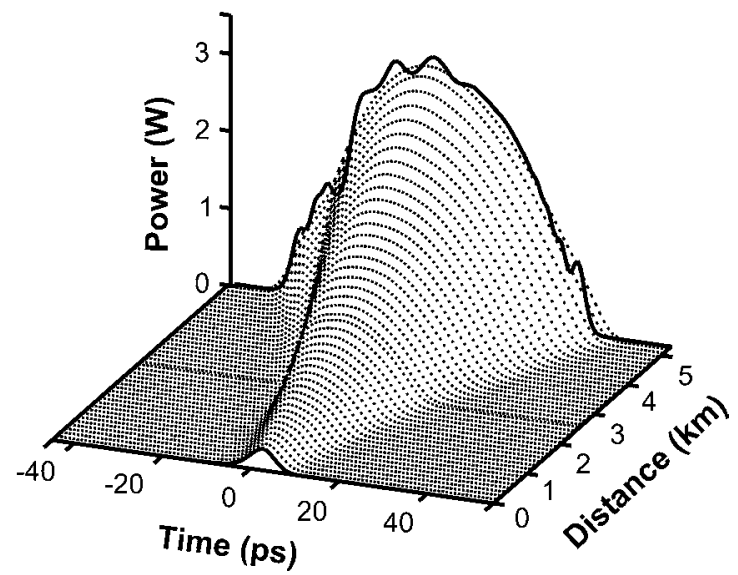

Fig. 6. Simulation (dashed lines) of parabolic pulse evolution over $5.3 \mathrm{~km}$ of NZ-DSF Raman amplifier. FROG retrievals of the intensity profiles of input and output pulses (solid lines).

pulses reveal that an asymmetry of the output pulses can also be observed in that case. So, other phenomena occur such as third-order dispersion and spectral asymmetry of the Raman gain (see Fig. 1). However, pump depletion associated to the walk-off effects is the main reason for this asymmetry, since the leading edge of the pulse experiences more gain than the trailing edge [see Fig. 2(a)].

Fig. 5 also shows the comparison between experimental data recorded at $1.3 \mathrm{~W}$ pump power with numerical results obtained by considering a pump power of only $0.95 \mathrm{~W}$ (crosses), and we note a very good agreement over several orders of magnitude. The dashed lines in Fig. 6 present three-dimensional (3-D) evolution plot of the numerical results showing the nonlinear transformation of the initial pulse toward a parabolic pulse during propagation through the 5.3-km NZ-DSF. The solid lines represent the experimental input and output pulse profiles. The difference of $27 \%$ between pump powers used in experiments and in simulations can be explained from polarization considerations. In particular, whereas the signal pulses are linearly polarized, the pump polarization is scrambled, so that the effective power acting in the amplification process is lower. Moreover, as the spectral width of the pulse is nearly $0.5 \mathrm{THz}$ and the propagation length is $5.3 \mathrm{~km}$, polarization mode dispersion (PMD) can affect the propagation. In another series of experiments (not shown here) performed with other fibers having slightly different parameters, we have observed that the effective power reduction depends sensibly on the fiber used, varying between $68 \%$ and $81 \%$. Note that these power reduction factors are in qualitative agreement with the reduction of the nonlinear parameter by a factor of $8 / 9$ which is commonly used in the case of random birefringence [13]. However, some further experiments would be needed to conclude to the validity of the theoretical model which predicts a reduction of the nonlinear parameter by a factor $8 / 9$. In particular, it would be of interest to measure the longitudinal evolution of the birefringence along the fiber. On the other hand, a recently developed vectorial formalism which includes the PMD effects in Raman fiber-based amplifiers could be used to consider these aspects in more detail [17], but is beyond the scope of this paper.

\section{Parabolic Pulse Properties}

Additional experiments were carried out to study in detail the dependence of the ouput parabolic pulse characteristics on input pulse duration and energy.

\section{A. Initial Pulse Duration Influence at Constant Energy}

We first study the influence of the initial pulse duration on the amplified output pulse. We thus consider two input pulses having different temporal widths but a constant 2.16-pJ energy. Fig. 7(a) shows the input pulses, with FWHM temporal widths of 14 and 7 ps, and peak powers of 120 and $230 \mathrm{~mW}$, respectively. The output amplified pulses are represented in Fig. 7(b).

We note that the 14- and 7-ps input pulses result in near-indentical output pulse characteristics (solid and dotted curves, respectively). From a theoretical viewpoint, in the context of a constant gain profile, it has been demonstrated that the asymptotic pulse characteristics are determined only by the incident pulse energy and the amplifier parameters, with the initial pulse shape determining only the evolution map toward this asymptotic solution [3]. As can be clearly seen from Fig. 7, our experimental results confirm remarquably this theoretical prediction.

\section{B. Influence of the Input Energy}

The dependence of the parabolic pulse characteristics on the energy of the input pulse has been experimentally studied by considering pulses of fixed 10-ps duration (FWHM) but with different energies of $0.32,1.3,2.16$, and $2.6 \mathrm{pJ}$. The retrieved intensity and chirp profiles obtained with the $0.32-, 1.3-$, and 2.6-pJ initial pulses are plotted in Fig. 8 and, in all cases, the output pulses clearly exhibit parabolic characteristics, that is a rapid falloff of the pulse wings and a linear chirp. The value of the chirp slope $C=11.810^{-3} \mathrm{THz}^{2}$ is independent of the initial pulse energy. Moreover, note that $C$ is also nearly independent of the initial shape [see Fig. 7(b)]. These results provide the first experimental confirmation of the theoretical predictions of Kruglov et al. [3] that the chirp slope of parabolic pulse in the case of a constant gain depends only on the gain and dispersion values.

To summarize our experimental results, Fig. 9 shows the evolution of temporal and spectral widths and peak power of the amplified pulses as a function of the input pulse energy. The increase of input pulse energy is associated with the simultaneous increase in the temporal and spectral widths and in the peak power of the amplified pulse. As can be seen in Fig. 9, a good agreement between experimental data (circles) and numerical simulations (solid lines) carried out with a pump power of $0.95 \mathrm{~W}$ is obtained.

\section{Propagation in a Normally Dispersive Fiber Without Gain}

Our goal here is to experimentally and numerically check the ability of a parabolic pulse to propagate in a normally dispersive fiber without gain, retaining its parabolic characteristics [4]. This essential property has already been outlined experimentally [5] with the propagation of a 2.6-ps, 12-nJ parabolic pulse in a $2-\mathrm{m}$ standard singlemode fiber at $1.06 \mu \mathrm{m}$.

Here, in order to generate the initial parabolic pulse, a 5-ps sech pulse is sent into the 5.3-km NZ-DSF and is amplified 

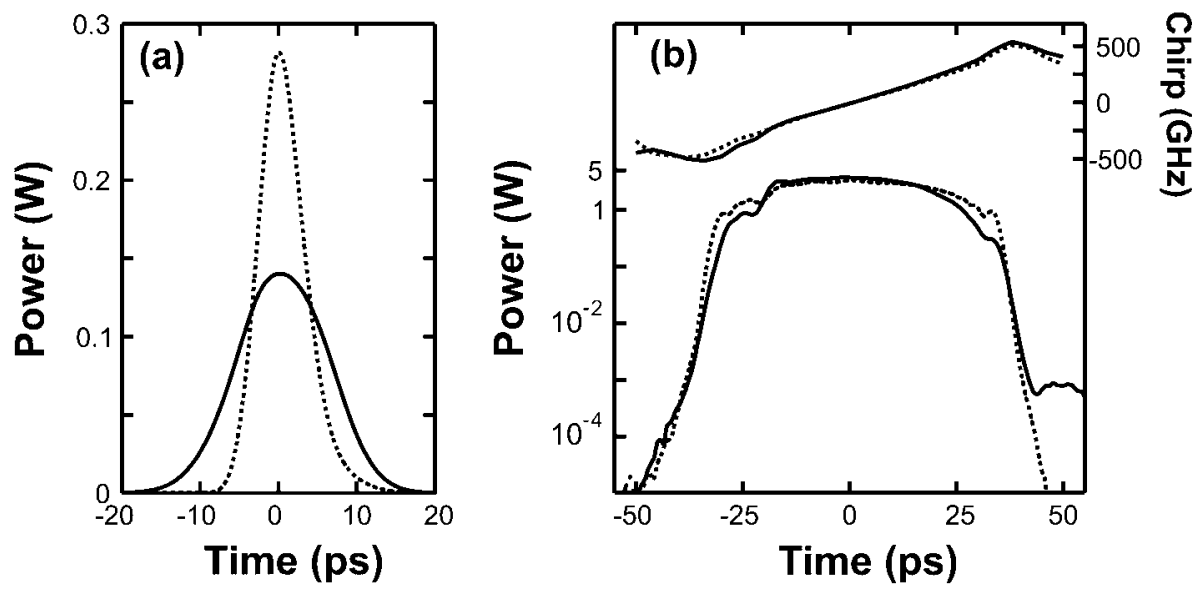

Fig. 7. Influence of the input intensity profile on the amplified pulse. Two particular pulses have been used with FWHM temporal widths of 14 ps (solid lines) and $7 \mathrm{ps}$ (circles). Intensity and chirp profiles of the input (a) and output (b) pulse retrieved from FROG measurements.

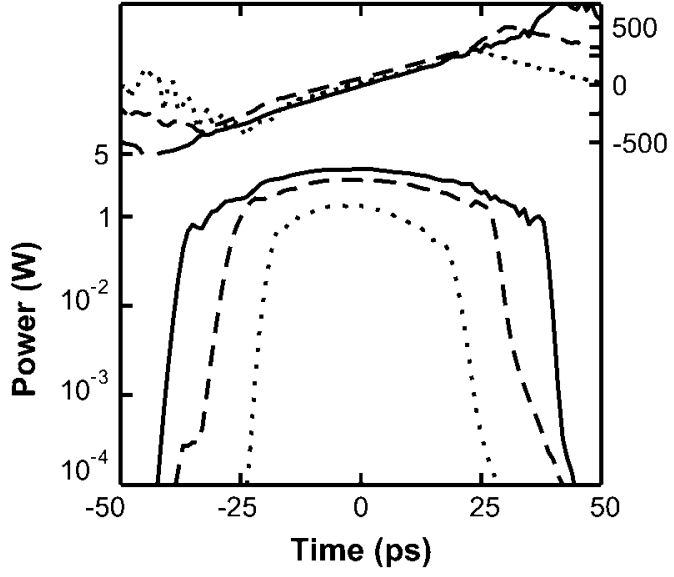

Fig. 8. Intensity and chirp profiles from FROG retrievals of output pulses obtained at different initial pulse energies: $0.32,1.3$, and $2.6 \mathrm{pJ}$ (dotted, dashed, and solid lines, respectively).

with a total gain of $18.8 \mathrm{~dB}$. The results of FROG characterization of the amplified pulse, reported in Fig. 10(a) (crosses), confirm well the generation of a parabolic pulse after propagation through the $5.3 \mathrm{~km}$ fiber. After filtering of the residual pump by a WDM coupler, the parabolic pulse is then sent into an 800-m normally dispersive NZ-DSF $\left(\beta_{2}=2.33 \times 10^{-3} \mathrm{ps}^{2}\right.$ $\mathrm{m}^{-1}$ ) without Raman pumping. The pulse at the output of the $800 \mathrm{~m}$ fiber was also characterized by the FROG technique. The results of this FROG characterization are plotted in Fig. 10(a) (circles). The retrieved intensity and chirp profiles of the pulse after $800 \mathrm{~m}$ of propagation are in excellent agreement with numerical results based (dashed line) on (1) without Raman gain and clearly exhibit parabolic features. One can conclude that, after a propagation distance of $800 \mathrm{~m}$ in a normally dispersive fiber, the parabolic characteristics are conserved.

We have also checked that this property was still verified for a larger distance of propagation. Due to the pulse broadening and the experimental limitations of our FROG device which is not adapted to characterize pulses longer than $100 \mathrm{ps}$, we were not able to carry out the experiment. However, as we have seen before, we can predict with high accuracy the results using the NLSE. The results of those numerical simulations are reported in Fig. 10(b) by circles. We display the parameter $\Delta \mathrm{C}$, defined as the difference of the chirp profile and its linear fit. The low

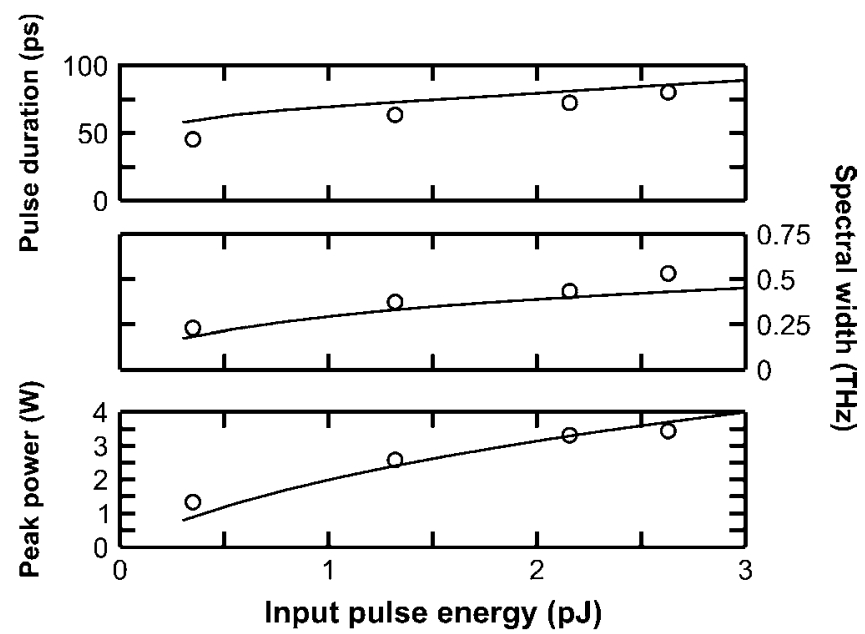

Fig. 9. Evolution of the parabolic pulse properties versus the input pulse energy. The output pulse temporal width corresponds to the width at $-20 \mathrm{~dB}$ of the peak power and the spectral width is the total width at half maximum. Experimental results are represented by circles and are given either by FROG trace retrievals (for the peak power or temporal width) or by direct measurements using an optical spectrum analyzer (spectral width). We note a good agreement with numerical results (solid lines).
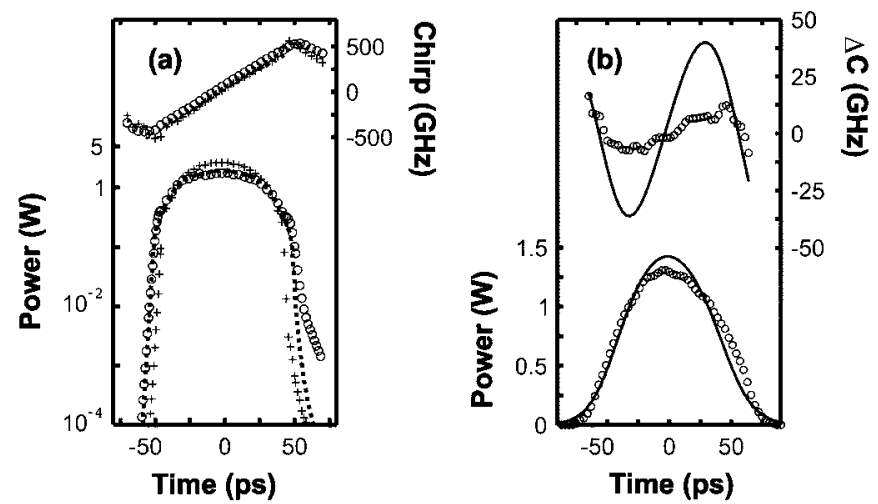

Fig. 10. (a) Intensity and chirp profiles obtained from FROG retrievals of a parabolic pulse before (crosses) and after (circles) propagation in a $800 \mathrm{~m}$ NZ-DSF fiber with $\beta_{2}=2.33 \times 10^{-3} \mathrm{ps} \mathrm{m}^{-1}$. Comparison with numerical simulations of the output pulse after $800 \mathrm{~m}$ of propagation (dashed line). (b) Intensity and $\triangle C$ profiles obtained from numerical propagation in $4 \mathrm{~km}$ of the same NZ-DSF fiber of two initial different pulses: the parabolic experimental pulse (circles) and a gaussian equivalent pulse (solid line).

value of $\Delta \mathrm{C}$ indicates that the chirp is still linear after $4 \mathrm{~km}$ of propagation. So, as the intensity profile also remains very close 

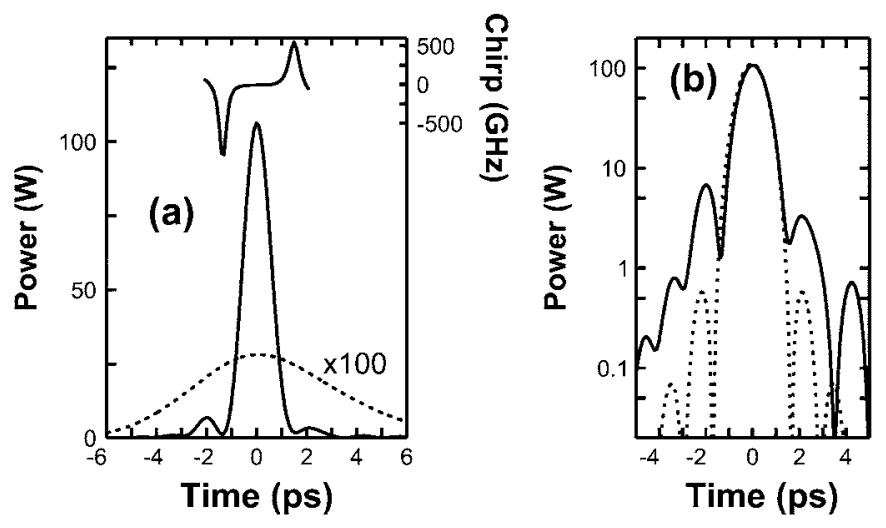

Fig. 11. (a) Intensity and chirp of compressed pulse (solid line) obtained after numerical linear chirp compensation of the output parabolic pulse described in Section III. Input pulse before the amplification process is plotted in dashes (magnification factor of 100 for the power). (b) Same results (solid line) on a logarithmic scale compared with compression of an exact parabolic pulse of the same energy and same temporal width (dashed line).

to a parabolic shape, we can conclude that after $4 \mathrm{~km}$ the pulse still exhibits the main characteristics of a parabolic pulse.

For comparison, we have studied the propagation of an equivalent linearly chirped gaussian pulse of the same energy. The temporal width and chirp parameter of the initial pulse have been choosen in order to minimize the difference with the experimental spectrum and autocorelation of the generated parabolic pulse described above. The results after propagation in the 4-km NZ-DSF are shown in Fig. 10(b) by solid lines. The intensity profile is still Gaussian, but the deviation from a linear fit of the chirp profile indicates that the chirp does not remain linear. This is consistent with the fact that, with the range of parameters used in these simulations, the main effect occuring during the propagation is nonlinear Kerr effect, leading to self-phase modulation of the pulse. This comparison highlights the particular behavior of a parabolic pulse which is able to maintain the linearity of its chirp even in presence of nonlinear effects.

\section{Compression of Parabolic Pulse}

The linearity of the parabolic pulse chirp would be expected to lead to efficient compression using only linear chirp compensation provided by, for example, a simple grating pair compressor. In this regard, an important feature of FROG characterization is that it permits the numerical computation of the expected compressed pulse characteristics based on the retrieved intensity and phase. For example, for the data presented in Fig. 5(a), numerical linear chirp compensation yields to the compressed pulse characteristics displayed in Fig. 11(a), which show negligible chirp variation across the pulse center. Here, the compressed pulse peak power is $102 \mathrm{~W}$ and the FWHM duration is $1.2 \mathrm{ps}$, which, when compared to the original input pulses from the fiber laser, represents a factor of 5.8 temporal compression and a factor of 450 increase in peak power. The compression quality is high, since the pedestals are pretty low and $92 \%$ of the energy is concentrated in the main peak.

In Fig. 11(b), we compare on a logarithmic scale the preceding results with a similar compression which could be achieved in the case of an exact parabolic pulse of the same energy and temporal width (dashed line). Note that even in this case, oscillations in the compressed pulse clearly remain and cannot be eliminated by using linear compression [18]. The origin of these oscillations comes from the compact support of parabolic pulses. We can conclude that results expected with a linear compression of the experimental parabolic pulses are very close to an optimal compression.

\section{CONCLUSION}

In this paper, we have presented the theoretical and experimental demonstration of parabolic pulse generation in Raman amplifier based on standard NZ-DSF and commercial devices adapted to telecommunication use. The use of SHG-FROG for pulse characterization allows the precise measurement of parabolic pulse features, such as the linear chirp and the rapid falloff in the wings of the intensity profile. We have been able to check some other essential properties of the parabolic pulses, such as the dependences on the duration and energy of the initial pulse, or their ability to propagate in a normally dispersive nonlinear fiber without loosing their properties.

\section{REFERENCES}

[1] K. Tamura and M. Nakazawa, "Pulse compression by nonlinear pulse evolution with reduced optical wave breaking in erbium-doped fiber amplifiers," Opt. Lett., vol. 21, pp. 68-70, 1996.

[2] V. I. Kruglov, A. C. Peacock, J. M. Dudley, and J. D. Harvey, "Self-similar propagation of high-power parabolic pulses in optical fiber amplifiers," Opt. Lett., vol. 25, pp. 1753-1755, 2000.

[3] - "Self-similar propagation of parabolic pulses in normal dispersion fiber amplifiers," J. Opt. Soc. Amer., vol. 19, pp. 461-469, 2002.

[4] D. Anderson, M. Desaix, M. Karlson, M. Lisak, and M. L. QuirogaTeixeiro, "Wave-breaking-free pulses in nonlinear optical fibers," J. Opt Soc. Amer. B, vol. 10, pp. 1185-1190, 1993.

[5] M. E. Fermann, V. I. Kruglov, B. C. Thomsen, J. M. Dudley, and J. D. Harvey, "Self-similar propagation and amplification of parabolic pulses in optical fibers," Phys. Rev. Lett., vol. 84, pp. 6010-6013, 2000.

[6] J. Limpert, T. Schreiber, T. Clausnitzer, K. Zöllner, H.-J. Fuchs, E.-B. Bley, H. Zellmer, and A. Tünnermann, "High power femtosecond Yb-doped fiber amplifier," Opt. Express, vol. 10, pp. 628-638, 2002.

[7] J. H. V. Price, W. Belardi, T. M. Monro, A. Malinowski, A. Piper, and D. J. Richardson, "Soliton transmission and supercontinuum generation in holey fiber, using a diode pumped Ytterbium fiber source," Opt. Express, vol. 10, pp. 382-387, 2002.

[8] F. Ö. Ilday, J. R. Buckley, W. G. Clark, and F. W. Wise, "Self-similar evolution of parabolic pulses in a laser," Phys. Rev. Lett, vol. 92, p. 213902, 2004.

[9] A. C. Peacock, N. G. R. Broderick, and T. M. Monro, "Numerical study of parabolic pulse generation in microstructured fiber Raman amplifiers," Opt. Commun., vol. 218, pp. 167-172, 2003.

[10] C. Billet, J. M. Dudley, C. Finot, and G. Millot, "Parabolic pulse generation at $1550 \mathrm{~nm}$ via Raman amplification in standard telecommunications grade dispersion shifted fiber," presented at the Conf. Lasers and ElectroOptics Europe, Munich, Germany, 2003, paper CL6-1-FRI.

[11] C. Finot, G. Millot, C. Billet, and J. M. Dudley, "Experimental generation of parabolic pulses via Raman amplification in optical fiber," Opt. Express, vol. 11, pp. 1547-1552, 2003.

[12] A. C. Peacock, R. J. Kruhlak, J. D. Harvey, and J. M. Dudley, "Solitary pulse propagation in high gain optical fiber amplifiers with normal group velocity dispersion," Opt. Commun., vol. 206, pp. 171-177, 2002.

[13] G. P. Agrawal, Nonlinear Fiber Optics, 3rd ed. San Fransisco, CA: Academic, 2001

[14] R. H. Stolen, J. P. Gordon, W. J. Tomlinson, and H. A. Haus, "Raman response function of silica-core fibers," J. Opt. Soc. Amer. B, vol. 6, pp. 1159-1166, 1989.

[15] C. Headley III and G. P. Agrawal, "Unified description of ultrafast stimulated Raman scattering in optical fibers," J. Opt. Soc. Amer. B, vol. 13, pp. 2170-2177, 1996.

[16] R. Trebino, Frequency-Resolved Optical Gating. The Measurement of Ultrashort Laser Pulses. Norwell, MA: Kluwer, 2000. 
[17] Q. Lin and G. P. Agrawal, "Vector theory of stimulated Raman scattering and its application to fiber-based Raman amplifiers," J. Opt. Soc. Amer. $B$, vol. 20, pp. 1616-1631, 2003.

[18] A. C. Peacock, "Self Similar amplification and propagation of parabolic pulses in optical fibers," M.S. thesis, Univ. , Auckland, New-Zealand, 2001. 